Research Article

\title{
The Feasibility of Homotopy Continuation Method for a Nonlinear Matrix Equation
}

\author{
Mengran Wang and Jing Li iD \\ School of Mathematics and Statistics, Shandong University, Weihai 264209, China \\ Correspondence should be addressed to Jing Li; xlijing@sdu.edu.cn \\ Received 15 November 2019; Accepted 4 March 2020; Published 24 April 2020 \\ Academic Editor: Nicolae Herisanu
}

Copyright (c) 2020 Mengran Wang and Jing Li. This is an open access article distributed under the Creative Commons Attribution License, which permits unrestricted use, distribution, and reproduction in any medium, provided the original work is properly cited.

In this paper, we discuss the feasibility of homotopy continuation method for the nonlinear matrix equations $X+\sum_{i=1}^{s} B_{i}^{*} X^{-1} B_{i}+$ $\sum_{i=s+1}^{m} B_{i}^{*} X^{t_{i}} B_{i}=I$ with $0<t_{i}<1$. This iterative method does not depend on a good initial approximation to the solution of matrix equation.

\section{Introduction}

In this paper, we consider the Hermitian positive definite (HPD) solutions of the nonlinear matrix equation:

$$
X+\sum_{i=1}^{s} B_{i}^{*} X^{-1} B_{i}+\sum_{i=s+1}^{m} B_{i}^{*} X^{t_{i}} \quad B_{i}=I, 0<t_{i}<1,
$$

where $B_{i}(i=1,2, \ldots, m)$ are $n \times n$ complex nonsingular matrices, $I$ is an $n \times n$ identity matrix, and $s \geq 1, m \geq 2$ are positive integers. Here, $B_{i}^{*}$ denotes the conjugate transpose of the matrix $B_{i}$.

The nonlinear matrix equations of (1) or some special cases are applicable to many fields such as nanoresearch, ladder networks, dynamic programming, control theory, stochastic filtering, and statistics [1-8].

Equation (1) is recognized as playing an important role in solving a system of linear equations. For example, in many physical calculations, one must solve the system of linear equation $M x=f$, where $x$ and $f$ are column vectors, and

$$
M=\left(\begin{array}{ccccc}
I & 0 & \cdots & 0 & A_{1} \\
0 & I & \cdots & 0 & A_{2} \\
\vdots & \vdots & \ddots & \vdots & \vdots \\
0 & 0 & \cdots & I & A_{m} \\
A_{1}^{*} & A_{2}^{*} & \cdots & A_{m}^{*} & I
\end{array}\right)
$$

arises in a finite difference approximation to an elliptic partial differential equation (for more information, refer to [2]). We can rewrite $M$ as $M=\widetilde{M}+D$, where

$$
\begin{aligned}
\widetilde{M} & =\left(\begin{array}{ccccccc}
X & \cdots & 0 & 0 & \cdots & 0 & A_{1} \\
\vdots & \ddots & \vdots & \vdots & & \vdots & \vdots \\
0 & \cdots & X & 0 & \cdots & 0 & A_{s} \\
0 & \cdots & 0 & X^{p_{s+1}} & \cdots & 0 & A_{s+1} \\
\vdots & \ddots & \vdots & \vdots & \ddots & \vdots & \vdots \\
0 & \cdots & 0 & 0 & \cdots & X^{p_{m}} & A_{m} \\
A_{1}^{*} & \cdots & A_{s}^{*} & A_{s+1}^{*} & \cdots & A_{m}^{*} & I
\end{array}\right), \\
\widetilde{D} & =\left(\begin{array}{ccccccc}
I-X & \cdots & 0 & 0 & \cdots & 0 & 0 \\
\vdots & \ddots & \vdots & \vdots & & \vdots & \vdots \\
0 & \cdots & I-X & 0 & \cdots & 0 & 0 \\
0 & \cdots & 0 & I-X^{p_{s+1}} & \cdots & 0 & 0 \\
\vdots & \ddots & \vdots & \vdots & \ddots & \vdots & \vdots \\
0 & \cdots & 0 & 0 & \cdots & I-X^{p_{m}} & 0 \\
0 & \cdots & 0 & 0 & \cdots & 0 & 0
\end{array}\right) .
\end{aligned}
$$


$\widetilde{M}$ can be factored as $\tilde{M}=\widetilde{M_{1}} \widetilde{M_{2}}$ if and only if $X$ is a solution of equation $X+\sum_{i=1}^{s} A_{i}^{*} X^{-1} A_{i}+\sum_{i=s+1}^{m} A_{i}^{*} X^{p_{i}}$ $A_{i}=I$, where

$$
\begin{aligned}
& \widetilde{M}_{1}=\left(\begin{array}{ccccccc}
I & \cdots & 0 & 0 & \cdots & 0 & 0 \\
\vdots & \ddots & \vdots & \vdots & & \vdots & \vdots \\
0 & \cdots & I & 0 & \cdots & 0 & 0 \\
0 & \cdots & 0 & I & \cdots & 0 & 0 \\
\vdots & \ddots & \vdots & \vdots & \ddots & \vdots & \vdots \\
0 & \cdots & 0 & 0 & \cdots & I & 0 \\
A_{1}^{*} X^{-1} & \cdots & A_{s}^{*} X^{-1} & A_{s+1}^{*} X^{p_{s+1}} & \cdots & A_{m}^{*} X^{p_{m}} & I
\end{array}\right), \\
& \widetilde{M}_{2}=\left(\begin{array}{ccccccc}
X & \cdots & 0 & 0 & \cdots & 0 & A_{1} \\
\vdots & \ddots & \vdots & \vdots & & \vdots & \vdots \\
0 & \cdots & X & 0 & \cdots & 0 & A_{s} \\
0 & \cdots & 0 & X^{p_{s+1}} & \cdots & 0 & A_{s+1} \\
\vdots & \ddots & \vdots & \vdots & \ddots & \vdots & \vdots \\
0 & \cdots & 0 & 0 & \cdots & X^{p_{m}} & A_{m} \\
0 & \cdots & 0 & 0 & \cdots & 0 & X
\end{array}\right) .
\end{aligned}
$$

Some special cases of matrix equation (1) and related matrix equations were studied on the solvability, numerical methods, and perturbation analysis by many scholars: $X+$ $A^{*} X^{-1} A=P \quad[9-12] ; X^{s}+A^{*} X^{-q} A=I \quad[13-17] ; X+A^{*}$ $X^{q} A=Q[18] ; X+\sum_{i=1}^{m} A_{i}^{*} X^{-1} A_{i}=I[19,20] ; X^{r}+\sum_{i=1}^{m}$ $A_{i}^{*} X^{\delta_{i}} A_{i}=I \quad[21,22] ; X+\sum_{i=1}^{m} A_{i}^{*} X^{-q} A_{i}=Q \quad[23] ; X+$ $\sum_{i=1}^{m} B_{i}^{*} X^{t_{i}} B_{i}=I$ [24]; and $X^{s} \pm A^{*} F(X) A=Q$ [25-29]. In addition, many homotopy approaches oriented toward engineering applications were investigated by many scholars: the homotopy analysis method (HAM) [30], the Optimal Homotopy Asymptotic Method (OHAM) [31], the Optimal Homotopy Perturbation Method (OHPM) [32], the homotopy asymptotic method [33], and so on.

According to our knowledge, the matrix equation (1) has not been treated explicitly in the literatures. The reason is that (1) does not always have a unique Hermitian positive definite solution. It is hard to find sufficient conditions for the existence of a unique Hermitian positive definite solution because the map $F(X)=I-\sum_{i=1}^{s} X^{-1} B_{i} B_{i}^{*}-\sum_{i=s+1}^{m}$ $B_{i}^{*} X^{t_{i}} B_{i}, 0<t_{i}<1$ is not monotonic. There are two difficulties for discussing the solvability and iterative method for the matrix equation (1). One is how to find a suitable set and some reasonable restrictions on the coefficient matrices ensuring this equation has a unique Hermitian positive definite solution in this set. The other one is how to find a reasonable expression of $F(X)-F(Y)$, which was important for discussing the feasibility of the homotopy continuation iterative method (see [34], for more details), which was not dependent on a good initial approximation to the solution of matrix equation.

In this paper, firstly, we derive necessary and sufficient conditions for the existence of Hermitian positive definite solutions to equation (1) in Section 3. Then, discuss the homotopy continuation methods for obtaining the unique Hermitian positive definite solution in Section 4.
The following notations are used throughout this paper. We denote by $\mathscr{C}^{n \times n}, \mathscr{H}^{n \times n}$, and $\mathscr{U}^{n \times n}$ the set of all $n \times n$ complex matrices, Hermitian matrices, and unitary matrices, respectively. For $A=\left(a_{1}, \ldots, a_{n}\right)=\left(a_{i j}\right) \in \mathscr{C}^{n \times n}$ and a matrix $B, A \otimes B=\left(a_{i j} B\right)$ is a Kronecker product, and vec $A$ is a vector defined by $\operatorname{vec} A=\left(a_{1}^{T}, \ldots, a_{n}^{T}\right)^{T}$. The symbol $\|\cdot\|$ stands for the spectral norm. We denote by $\lambda_{i}(M)$ the eigenvalues of $M$, by $\lambda_{1}(M)$ and $\lambda_{n}(M)$ the maximal and minimal eigenvalues of $M$, respectively. For $X, Y \in \mathscr{H}^{n \times n}$, we write $X \geq Y(X>Y)$, if $X-Y$ is a Hermitian positive semidefinite (definite) matrix. For $A, B \in \mathscr{H}^{n \times n}$, the sets $[A, B]$ and $(A, B]$ are defined by $[A, B]=\left\{X \in \mathscr{H}^{n \times n} \mid A \leq X \leq B\right\}$ and $(A, B]=\left\{X \in \mathscr{H}^{n \times n} \mid A<X \leq B\right\}$.

\section{Preliminaries}

In this section, we present some lemmas that will be needed to develop this paper.

Lemma 1 (see [35]). If $A \geq B>0$ and $0 \leq \gamma \leq 1$, then $A^{\gamma} \geq B^{\gamma}$.

Lemma 2 (see [24], Lemma 3.3). For every Hermitian positive definite matrix $X$ and $0<t<1$, it yields that $X^{t}=\sin t \pi / \pi \int_{0}^{\infty} X(\lambda I+X)^{-1} \lambda^{t-1} d \lambda$.

Lemma 3 (see [36], Theorem 1.9.1). Let $A \in \mathscr{C}^{m \times n}$, $B \in \mathscr{C}^{p \times q}, C \in \mathscr{C}^{n \times k}$, and $D \in \mathscr{C}^{q \times r}$. Then,

$$
\begin{aligned}
& (A \otimes B)(C \otimes D)=(A C) \otimes(B D), \\
& (A \otimes B)^{*}=A^{*} \otimes B^{*}
\end{aligned}
$$

Lemma 4 (see [36], Lemma 1.9.1). Let $A \in \mathscr{C}^{l \times m}$, $X \in \mathscr{C}^{m \times n}$, and $B \in \mathscr{C}^{n \times k}$. Then,

$$
\operatorname{vec}(A X B)=\left(B^{T} \otimes A\right) \cdot \operatorname{vec} X .
$$

Lemma 5 (see [37], Theorem 6.19). Let $A \in \mathscr{C}^{m \times m}$ and $B \in \mathscr{C}^{n \times n}$ with eigenvalues $\lambda_{i}$ and $\mu_{j}, i=1,2, \ldots, m$, $j=1,2, \ldots, n$, respectively. Then, the eigenvalues of $A \otimes B$ are $\lambda_{i} \mu_{j}, i=1,2, \ldots, m$ and $j=1,2, \ldots, n$.

Lemma 6 (see [24], Lemma 3.2). Suppose that $m \geq 1,0<t<1$, and $(m t /(m t+1))<x, y<1$. Then,

$$
0<f(x, y, t)=\frac{\sqrt{(1-x)(1-y)}\left(x^{t}-y^{t}\right)}{(x-y) x^{(t / 2)} y^{(t / 2)}}<\frac{1}{m} \text {. }
$$

\section{Hermitian Positive Definite Solutions}

In this section, some sufficient and necessary conditions for the existence and uniqueness of HPD solution of (1) are derived.

Theorem 1. (1) has a HPD solution if and only if there exist $Q_{i} \in \mathscr{C}^{n \times n}, i=1,2, \ldots, m, P \in \mathcal{U}^{n \times n}$, and diagonal matrices $\Gamma, \Lambda>0$ such that 


$$
B_{i}= \begin{cases}P^{*} \Gamma^{(1 / 2)} Q_{i} \Lambda P, & i=1,2, \ldots, s, \\ P^{*} \Gamma^{-\left(t_{i} / 2\right)} Q_{i} \Lambda P, & i=s+1, s+2, \ldots, m,\end{cases}
$$

where $\Lambda^{2}+\Gamma=I$ and $\sum_{i=1}^{m} Q_{i}^{*} Q_{i}=I$. In this case, $Y=P^{*} \Gamma P$ is a HPD solution of (1).

Proof. Assume $Y$ is an HPD solution of (1). According to the spectral decomposition theorem, we have that there exists $P \in \mathscr{U}^{n \times n}$ and a diagonal matrix $\Gamma>0$ such that $Y=P^{*} \Gamma P$. Then, (1) can be expressed as

$$
P^{*} \Gamma P+\sum_{i=1}^{s} B_{i}^{*} P^{*} \Gamma^{-1} P B_{i}+\sum_{i=s+1}^{m} B_{i}^{*} P^{*} \Gamma^{t_{i}} P B_{i}=I .
$$

Multiplying the left side of (8) by $P$ and the right side by $P^{*}$, we obtain

$$
\sum_{i=1}^{s} P B_{i}^{*} P^{*} \Gamma^{-1} P B_{i} P^{*}+\sum_{i=s+1}^{m} P B_{i}^{*} P^{*} \Gamma^{t_{i}} P B_{i} P^{*}=I-\Gamma .
$$

Recall that $B_{i}(i=1,2, \ldots, m)$ are nonsingular matrices. Then,

$$
0<\Gamma<I .
$$

Therefore, we can rewrite (10) as

$$
\begin{aligned}
& \sum_{i=1}^{s}(I-\Gamma)^{-(1 / 2)} P B_{i}^{*} P^{*} \Gamma^{-1} P B_{i} P^{*}(I-\Gamma)^{-(1 / 2)} \\
& +\sum_{i=s+1}^{m}(I-\Gamma)^{-(1 / 2)} P B_{i}^{*} P^{*} \Gamma^{t_{i}} P B_{i} P^{*}(I-\Gamma)^{-(1 / 2)}=I .
\end{aligned}
$$

Let

$$
\begin{aligned}
\Lambda & =(I-\Gamma)^{(1 / 2)}, \\
Q_{i} & = \begin{cases}\Gamma^{-(1 / 2)} P B_{i} P^{*} \Lambda^{-1}, & i=1,2, \ldots, s, \\
\Gamma^{\left(t_{i} / 2\right)} P B_{i} P^{*} \Lambda^{-1}, & i=s+1, s+2, \ldots, m .\end{cases}
\end{aligned}
$$

It is easy to verify that $\Gamma+\Lambda^{2}=I$ and

$$
B_{i}= \begin{cases}P^{*} \Gamma^{(1 / 2)} Q_{i} \Lambda P, & i=1,2, \ldots, s, \\ P^{*} \Gamma^{-\left(t_{i} / 2\right)} Q_{i} \Lambda P, & i=s+1, s+2, \ldots, m .\end{cases}
$$

By (11), we obtain $\sum_{i=1}^{m} Q_{i}^{*} Q_{i}=I$.

Conversely, assume there exist $P \in \mathscr{U}^{n \times n}, Q_{i} \in \mathscr{C}^{n \times n}$, $\sum_{i=1}^{m} Q_{i}^{*} Q_{i}=I$, and diagonal matrices $\Gamma, \Lambda>0, \Lambda^{2}+\Gamma=I$ such that

$$
B_{i}= \begin{cases}P^{*} \Gamma^{(1 / 2)} Q_{i} \Lambda P, & i=1,2, \ldots, s, \\ P^{*} \Gamma^{-\left(t_{i} / 2\right)} Q_{i} \Lambda P, & i=s+1, s+2, \ldots, m .\end{cases}
$$

Let $Y=P^{*} \Gamma P$ such that

$$
\begin{aligned}
Y & +\sum_{i=1}^{s} B_{i}^{*} Y^{-1} B_{i}+\sum_{i=s+1}^{m} B_{i}^{*} X^{t_{i}} B_{i} \\
& =P^{*} \Gamma P+\sum_{i=1}^{s} P^{*} \Lambda^{*} Q_{i}^{*} \Gamma^{(1 / 2)} P\left(P^{*} \Gamma P\right)^{-1} P^{*} \Gamma^{\left(t_{i} / 2\right)} Q_{i} \Lambda P \\
& +\sum_{i=s+1}^{m} P^{*} \Lambda^{*} Q_{i}^{*} \Gamma^{-\left(t_{i} / 2\right)} P\left(P^{*} \Gamma P\right)^{t_{i}} P^{*} \Gamma^{-\left(t_{i} / 2\right)} Q_{i} \Lambda P \\
& =P^{*} \Gamma P+\sum_{i=1}^{m} P^{*} \Lambda Q_{i}^{*} Q_{i} \Lambda P=P^{*}\left(\Gamma+\Lambda^{2}\right) P=I,
\end{aligned}
$$

which means $Y$ is an HPD solution of (1).

Theorem 2. If $\lambda_{1}\left(\sum_{i=1}^{m} B_{i}^{*} B_{i}\right)<\left(m /(m+1)^{2}\right)$, then (1) has a unique HPD solution $X$ on $[(m /(m+1)) I, I]$.

Proof.

Step 1. We will prove that (1) has a HPD solution on $[(m /(m+1)) I, I]$ under the assumption $\lambda_{1}\left(\sum_{i=1}^{m} B_{i}^{*} B_{i}\right)$ $<\left(m /(m+1)^{2}\right)$.

Let $\Omega=[(m /(m+1)) I, I]$. Define

$$
F(X)=I-\sum_{i=1}^{s} B_{i}^{*} X^{-1} B_{i}-\sum_{i=s+1}^{m} B_{i}^{*} X^{t_{i}} B_{i}, X \in \Omega .
$$

Obviously, $\Omega$ is a bounded convex closed set and $F$ is continuous on $\Omega$.

Note that $0<t_{i}<1$. For any $X \in \Omega$, it follows from Lemma 1 that $(m /(m+1))^{t_{i}} I \leq X^{t_{i}} \leq I$, which implies

$$
\begin{aligned}
I & \geq F(X) \geq I-\sum_{i=1}^{s}\left(\frac{(m+1)}{m}\right) B_{i}^{*} B_{i}-\sum_{i=s+1}^{m} B_{i}^{*} B_{i} \\
& \geq I-\left(\frac{(m+1)}{m}\right) \sum_{i=1}^{m} B_{i}^{*} B_{i} .
\end{aligned}
$$

Recall that $\lambda_{1}\left(\sum_{i=1}^{m} B_{i}^{*} B_{i}\right)<\left(m /(m+1)^{2}\right)$, then $I \geq F(X) \geq$ $(m /(m+1)) I$. That is, $F(X) \subseteq \Omega$. By Brouwer's fixed point theorem, the map $F$ has a fixed point $X \in \Omega$, which is a HPD solution of (1).

Step 2. We will prove that if (1) has a HPD solution on $[(m /(m+1)) I, I]$, then the HPD solution is unique.

If $Y_{1}$ is a HPD solution of (1), according to Lemma 3.1, there exist $P_{1} \in \mathscr{U}^{n \times n}, Q_{i} \in \mathscr{C}^{n \times n}, i=1,2, \ldots, m$, and diagonal matrices $\Gamma_{1}, \Lambda_{1}>0$ such that

$$
B_{i}= \begin{cases}P_{1}^{*} \Gamma_{1}^{(1 / 2)} Q_{i} \Lambda_{1} P_{1}, & i=1,2, \ldots, s, \\ P_{1}^{*} \Gamma_{1}^{-\left(t_{i} / 2\right)} Q_{i} \Lambda_{1} P_{1}, & i=s+1, s+2, \ldots, m,\end{cases}
$$

where

$$
\begin{aligned}
\sum_{i=1}^{m} Q_{i}^{*} Q_{i} & =I, \\
\Lambda_{1}^{2}+\Gamma_{1} & =I .
\end{aligned}
$$


In this case, $Y_{1}=P_{1}^{*} \Gamma_{1} P_{1}$, where $\Gamma_{1}=\operatorname{diag}\left(\lambda_{11}, \lambda_{12}\right.$, $\left.\ldots, \lambda_{1 n}\right)$ with $\left\{\lambda_{1 j}\right\}$ the eigenvalues of $Y_{1}$. Similarly, if $Y_{2}$ is a HPD solution of (1), then there exist $P_{2} \in \mathscr{U}^{n \times n}, U_{i} \in$ $\mathscr{C}^{n \times n}, i=1,2, \ldots, m$, and diagonal matrices $\Gamma_{2}, \Lambda_{2}>0$ such that

$$
B_{i}= \begin{cases}P_{2}^{*} \Gamma_{2}^{(1 / 2)} U_{i} \Lambda_{2} P_{2}, & i=1,2, \ldots, s, \\ P_{2}^{*} \Gamma_{2}^{-\left(t_{i} / 2\right)} U_{i} \Lambda_{2} P_{2}, & i=s+1, s+2, \ldots, m,\end{cases}
$$

where

$$
\begin{aligned}
\sum_{i=1}^{m} U_{i}^{*} U_{i} & =I, \\
\Lambda_{2}^{2}+\Gamma_{2} & =I .
\end{aligned}
$$

In this case, $Y_{2}=P_{2}^{*} \Gamma_{2} P_{2}$, where $\Gamma_{2}=\operatorname{diag}\left(\lambda_{21}, \lambda_{22}, \ldots, \lambda_{2 n}\right)$ with $\left\{\lambda_{2 j}\right\}$ the eigenvalues of. According to $Y_{2}$ in Lemma 2, we have

$$
\begin{aligned}
Y_{1}-Y_{2}= & \sum_{i=1}^{s} B_{i}^{*}\left(Y_{2}^{-1}-Y_{1}^{-1}\right) B_{i}+\sum_{i=s+1}^{m} B_{i}^{*}\left(Y_{2}^{t_{i}}-Y_{1}^{t_{i}}\right) B_{i} \\
= & \sum_{i=1}^{s} B_{i}^{*} Y_{2}^{-1}\left(Y_{1}-Y_{2}\right) Y_{1}^{-1} B_{i}+\sum_{i=s+1}^{m} \frac{B_{i}^{*} \sin t_{i} \pi}{\pi} \int_{0}^{\infty}\left[Y_{2}\left(\lambda I+Y_{2}\right)^{-1}-Y_{1}\left(\lambda I+Y_{1}\right)^{-1}\right] \lambda^{t_{i}-1} B_{i} \mathrm{~d} \lambda \\
= & \sum_{i=1}^{s} B_{i}^{*} Y_{2}^{-1}\left(Y_{1}-Y_{2}\right) Y_{1}^{-1} B_{i}-\sum_{i=s+1}^{m} \frac{B_{i}^{*} \sin t_{i} \pi}{\pi} \int_{0}^{\infty}\left(Y_{1}-Y_{2}\right)\left(\lambda I+Y_{1}\right)^{-1} \lambda^{t_{i}-1} B_{i} \mathrm{~d} \lambda \\
& +\sum_{i=s+1}^{m} \frac{B_{i}^{*} \sin t_{i} \pi}{\pi} \int_{0}^{\infty} Y_{2}\left(\lambda I+Y_{2}\right)^{-1}\left(Y_{1}-Y_{2}\right)\left(\lambda I+Y_{1}\right)^{-1} \lambda^{t_{i}-1} B_{i} \mathrm{~d} \lambda .
\end{aligned}
$$

By

$$
\left(\lambda I+Y_{i}\right)^{-1}=\left(\lambda I+P_{i}^{*} \Gamma_{i} P_{i}\right)^{-1}=P_{i}^{-1}\left(\lambda I+\Gamma_{i}\right)^{-1} P_{i}, \quad i=1,2,
$$

we have

$$
\begin{aligned}
Y_{1}-Y_{2}= & \sum_{i=1}^{s}\left(P_{2}^{*} \Gamma_{2}^{1 / 2} U_{i} \Lambda_{2} P_{2}\right)^{*} P_{2}^{-1} \Gamma_{2}^{-1} P_{2}\left(Y_{1}-Y_{2}\right) P_{1}^{*} \Gamma_{1}^{-1} P_{1}\left(P_{1}^{*} \Gamma_{1}^{1 / 2} Q_{i} \Lambda_{1} P_{1}\right) \\
& -\sum_{i=s+1}^{m} \frac{\sin t_{i} \pi}{\pi} \int_{0}^{\infty} P_{2}^{*} \Lambda_{2} U_{i}^{*} \Gamma_{2}^{-\frac{t_{i}}{2}} P_{2}\left(Y_{1}-Y_{2}\right) P_{1}^{*}\left(\lambda I+\Gamma_{1}\right)^{-1} \Gamma_{1}^{-t_{i} / 2} Q_{i} \Lambda_{1} P_{1} \lambda^{t_{i}-1} d \lambda \\
& +\sum_{i=s+1}^{m} \frac{\sin t_{i} \pi}{\pi} \int_{0}^{\infty} P_{2}^{*} \Lambda_{2} U_{i}^{*} \Gamma_{2}^{1-\frac{t_{i}}{2}}\left(\lambda I+\Gamma_{2}\right)^{-1} P_{2}\left(Y_{1}-Y_{2}\right) P_{1}^{*}\left(\lambda I+\Gamma_{1}\right)^{-1} \Gamma_{1}^{-t_{i} / 2} Q_{i} \Lambda_{1} P_{1} \lambda^{t_{i}-1} d \lambda
\end{aligned}
$$

Let

$$
W=P_{2}\left(Y_{1}-Y_{2}\right) P_{1}^{*}
$$

Then, (24) can be expressed as

$$
\begin{aligned}
W= & \sum_{i=1}^{s} \Lambda_{2} U_{i}^{*} \Gamma_{2}^{-(1 / 2)} W \Gamma_{1}^{-(1 / 2)} Q_{i} \Lambda_{1}-\sum_{i=s+1}^{m} \frac{\sin t_{i} \pi}{\pi} \int_{0}^{\infty} \Lambda_{2} U_{i}^{*} \Gamma_{2}^{-\left(t_{i} / 2\right)} W\left(\lambda I+\Gamma_{1}\right)^{-1} \Gamma_{1}^{-\left(t_{i} / 2\right)} Q_{i} \Lambda_{1} \lambda^{t_{i}-1} \mathrm{~d} \lambda \\
& +\sum_{i=s+1}^{m} \frac{\sin t_{i} \pi}{\pi} \int_{0}^{\infty} \Lambda_{2} U_{i}^{*} \Gamma_{2}^{1-\left(t_{i} / 2\right)}\left(\lambda I+\Gamma_{2}\right)^{-1} W\left(\lambda I+\Gamma_{1}\right)^{-1} \Gamma_{1}^{-\left(t_{i} / 2\right)} Q_{i} \Lambda_{1} \lambda^{t_{i}-1} \mathrm{~d} \lambda .
\end{aligned}
$$


By (26), Lemmas 3 and 4, we have that

$$
\begin{aligned}
\operatorname{vec} W= & \sum_{i=1}^{s}\left[\Gamma_{1}^{-(1 / 2)} Q_{i} \Lambda_{1}\right]^{T} \otimes\left[\Lambda_{2} U_{i}^{*} \Gamma_{2}^{-(1 / 2)}\right] \cdot \operatorname{vec} W \\
& -\sum_{i=s+1}^{m} \frac{\sin t_{i} \pi}{\pi} \int_{0}^{\infty}\left[\left(\lambda I+\Gamma_{1}\right)^{-1} \Gamma_{1}^{-\left(t_{i} / 2\right)} Q_{i} \Lambda_{1}\right]^{T} \otimes\left(\Lambda_{2} U_{i}^{*} \Gamma_{2}^{-\left(t_{i} / 2\right)}\right) \lambda^{t_{i}-1} \mathrm{~d} \lambda \operatorname{vec} W \\
& +\sum_{i=s+1}^{m} \frac{\sin t_{i} \pi}{\pi} \int_{0}^{\infty}\left[\left(\lambda I+\Gamma_{1}\right)^{-1} \Gamma_{1}^{-\left(t_{i} / 2\right)} Q_{i} \Lambda_{1}\right]^{T} \otimes\left[\Lambda_{2} U_{i}^{*} \Gamma_{2}^{1-\left(t_{i} / 2\right)}\left(\lambda I+\Gamma_{2}\right)^{-1}\right] \lambda^{t_{i}-1} \mathrm{~d} \lambda \operatorname{vec} W \\
= & \sum_{i=1}^{s}\left(\Lambda_{1} \otimes \Lambda_{2}\right)\left(Q_{i}^{T} \otimes U_{i}^{*}\right)\left(\Gamma_{1}^{(1 / 2)} \otimes \Gamma_{2}^{(1 / 2)}\right) \cdot \operatorname{vec} W \\
& -\sum_{i=s+1}^{m} \frac{\sin t_{i} \pi}{\pi}\left(\Lambda_{1} \otimes \Lambda_{2}\right)\left(Q_{i}^{T} \otimes U_{i}^{*}\right)\left(\Gamma_{1}^{-\left(t_{i} / 2\right)} \otimes \Gamma_{2}^{-\left(t_{i} / 2\right)}\right) \int_{0}^{\infty}\left[\left(\lambda I+\Gamma_{1}\right)^{-1} \otimes I\right] \lambda^{t_{i}-1} \mathrm{~d} \lambda \operatorname{vec} W \\
& +\sum_{i=s+1}^{m} \frac{\sin t_{i} \pi}{\pi}\left(\Lambda_{1} \otimes \Lambda_{2}\right)\left(Q_{i}^{T} \otimes U_{i}^{*}\right)\left(\Gamma_{1}^{-\left(t_{i} / 2\right)} \otimes \Gamma_{2}^{1-\left(t_{i} / 2\right)}\right) \int_{0}^{\infty}\left(\lambda I+\Gamma_{1}\right)^{-1} \otimes\left(\lambda I+\Gamma_{2}\right)^{-1} \lambda^{t_{i}-1} \mathrm{~d} \lambda \mathrm{vec} W
\end{aligned}
$$

Assume that

$$
\begin{aligned}
& \Lambda_{1}=\operatorname{diag}\left(\sigma_{11}, \sigma_{12}, \ldots, \sigma_{1 n}\right), \\
& \Lambda_{2}=\operatorname{diag}\left(\sigma_{21}, \sigma_{22}, \ldots, \sigma_{2 n}\right) .
\end{aligned}
$$

$$
0<\sigma_{1 j}=\sqrt{1-\lambda_{1 j}}<1, \quad 0<\sigma_{2 j}=\sqrt{1-\lambda_{2 j}}<1, \quad j=1,2, \ldots, n .
$$

Let

$$
\begin{aligned}
B & =\Lambda_{1} \otimes \Lambda_{2}, J_{i}=Q_{i}^{T} \otimes U_{i}^{*}, E=\Gamma_{1}^{(1 / 2)} \otimes \Gamma_{2}^{(1 / 2)} i=1,2, \ldots, m \\
C_{i} & =\left(\Gamma_{1}^{-\left(t_{i} / 2\right)} \otimes \Gamma_{2}^{-\left(t_{i} / 2\right)}\right) \frac{\sin t_{i} \pi}{\pi} \int_{0}^{\infty}\left[\left(\lambda I+\Gamma_{1}\right)^{-1} \otimes I\right] \lambda^{t_{i}-1} \mathrm{~d} \lambda, \quad i=s+1, s+2, \ldots, m, \\
D_{i} & =\left(\Gamma_{1}^{-\left(t_{i} / 2\right)} \otimes \Gamma_{2}^{1-\left(t_{i} / 2\right)}\right) \frac{\sin t_{i} \pi}{\pi} \int_{0}^{\infty}\left(\lambda I+\Gamma_{1}\right)^{-1} \otimes\left(\lambda I+\Gamma_{2}\right)^{-1} \lambda^{t_{i}-1} \mathrm{~d} \lambda, \quad i=s+1, s+2, \ldots, m .
\end{aligned}
$$

Then, (27) can be expressed as

$\operatorname{vec} W+B\left[\sum_{i=s+1}^{m} J_{i}\left(C_{i}-D_{i}\right)-\sum_{i=1}^{s} J_{i} E\right] \cdot \operatorname{vec} W=0$. 
By Lemma 5, we have

$$
\begin{aligned}
& B=\Lambda_{1} \otimes \Lambda_{2}=\operatorname{diag}\left(\sigma_{1 l} \cdot \sigma_{2 j}\right)_{n^{2} \times n^{2}}, \quad l, j=1,2, \ldots, n, \\
& C_{i}=\left(\Gamma_{1}^{-\left(t_{i} / 2\right)} \otimes \Gamma_{2}^{-\left(t_{i} / 2\right)}\right) \cdot \frac{\sin t_{i} \pi}{\pi} \int_{0}^{\infty}\left[\left(\lambda I+\Gamma_{1}\right)^{-1} \otimes I\right] \lambda^{t_{i}-1} \mathrm{~d} \lambda \\
& =\operatorname{diag}\left(\lambda_{1 l}^{-\left(t_{i} / 2\right)} \cdot \lambda_{2 j}^{-\left(t_{i} / 2\right)} \cdot \frac{\sin t_{i} \pi}{\pi} \int_{0}^{\infty}\left(\lambda+\lambda_{1 l}\right)^{-1} \lambda^{t_{i}-1} \mathrm{~d} \lambda\right)_{n^{2} \times n^{2}} \\
& =\operatorname{diag}\left(\lambda_{1 l}^{-\left(t_{i} / 2\right)} \cdot \lambda_{2 j}^{-\left(t_{i} / 2\right)} \cdot \lambda_{1 l}^{t_{i}-1}\right)_{n^{2} \times n^{2}} \\
& =\operatorname{diag}\left(\lambda_{1 l}^{\left(t_{i} / 2\right)-1} \cdot \lambda_{2 j}^{-\left(t_{i} / 2\right)}\right)_{n^{2} \times n^{2}}, \quad i=(s+1),(s+2), \ldots, m, \quad l, j=1,2, \ldots, n, \\
& D_{i}=\left(\Gamma_{1}^{-\left(t_{i} / 2\right)} \otimes \Gamma_{2}^{1-\left(t_{i} / 2\right)}\right) \cdot \frac{\sin t_{i} \pi}{\pi} \int_{0}^{\infty}\left(\lambda I+\Gamma_{1}\right)^{-1} \otimes\left(\lambda I+\Gamma_{2}\right)^{-1} \lambda^{t_{i}-1} \mathrm{~d} \lambda \\
& =\operatorname{diag}\left(\lambda_{1 l}^{-\left(t_{i} / 2\right)} \cdot \lambda_{2 j}^{1-t_{i} / 2} \cdot \frac{\sin t_{i} \pi}{\pi} \int_{0}^{\infty}\left(\lambda+\lambda_{1 l}\right)^{-1}\left(\lambda+\lambda_{2 j}\right)^{-1} \lambda^{t_{i}-1} \mathrm{~d} \lambda\right)_{n^{2} \times n^{2}} \\
& =\operatorname{diag}\left(\frac{\lambda_{1 l}^{-\left(t_{i} / 2\right)} \cdot \lambda_{2 j}^{1-\left(t_{i} / 2\right)}\left(\lambda_{1 l}^{t_{i}-1}-\lambda_{2 j}^{t_{i}-1}\right)}{\lambda_{2 j}-\lambda_{1 l}}\right)_{n^{2} \times n^{2}}, \quad i=(s+1),(s+2), \ldots, m, \quad l, j=1,2, \ldots, n \\
& E=\Gamma_{1}^{-(1 / 2)} \otimes \Gamma_{2}^{-(1 / 2)}=\operatorname{diag}\left(\lambda_{1 l}^{-(1 / 2)} \cdot \lambda_{2 j}^{-(1 / 2)}\right)_{n^{2} \times n^{2}}, \quad l, j=1,2, \ldots, n .
\end{aligned}
$$

It follows that

$$
\begin{aligned}
C_{i}-D_{i} & =\operatorname{diag}\left(\lambda_{1 l}^{\left(t_{i} / 2\right)-1} \cdot \lambda_{2 j}^{-\left(t_{i} / 2\right)}-\frac{\lambda_{1 l}^{-\left(t_{i} / 2\right)} \cdot \lambda_{2 j}^{1-\left(t_{i} / 2\right)}\left(\lambda_{1 l}^{t_{i}-1}-\lambda_{2 j}^{t_{i}-1}\right)}{\lambda_{2 j}-\lambda_{1 l}}\right)_{n^{2} \times n^{2}} \\
& =\operatorname{diag}\left(\frac{\lambda_{1 l}^{t_{i}}-\lambda_{2 j}^{t_{i}}}{\left(\lambda_{1 l}-\lambda_{2 j}\right) \lambda_{1 l}^{\left(t_{i} / 2\right)} \lambda_{2 j}^{\left(t_{i} / 2\right)}}\right)_{n^{2} \times n^{2}}, \quad i=(s+1),(s+2), \ldots, m .
\end{aligned}
$$

Note that $B$ is nonsingular. Multiplying the left side of (31) by $B^{-1}$, we have

$$
\begin{aligned}
& B^{-1} \mathrm{vec} W+\left[\sum_{i=s+1}^{m} J_{i}\left(C_{i}-D_{i}\right)-\sum_{i=1}^{s} J_{i} E\right] \cdot \operatorname{vec} W \\
& =\left[I+\sum_{i=s+1}^{m} J_{i}\left(C_{i}-D_{i}\right) B-\sum_{i=1}^{s} J_{i} E B\right] B^{-1} \cdot \operatorname{vec} W=0 .
\end{aligned}
$$

A combination of (30) and Lemma 3 gives

$$
\begin{aligned}
J_{i}^{*} J_{i} & =\left(Q_{i}^{T} \otimes U_{i}^{*}\right)^{*}\left(Q_{i}^{T} \otimes U_{i}^{*}\right)=\left(\bar{Q}_{i} \otimes U_{i}\right)\left(Q_{i}^{T} \otimes U_{i}^{*}\right) \\
& =\left(\bar{Q}_{i} Q_{i}^{T}\right) \otimes\left(U_{i} U_{i}^{*}\right)=\left(\overline{Q_{i} Q_{i}^{*}}\right) \otimes\left(U_{i} U_{i}^{*}\right) .
\end{aligned}
$$

It follows (19), (21), and Lemma 5 that $0<\left\|J_{i}\right\| \leq 1$. Therefore,

$$
\left\|\sum_{i=(s+1)}^{m} J_{i}\left(C_{i}-D_{i}\right) B\right\| \leq \sum_{i=(s+1)}^{m}\left\|\left(C_{i}-D_{i}\right) B\right\|,\left\|\sum_{i=1}^{s} J_{i} E B\right\| \leq \sum_{i=1}^{s}\|E B\| .
$$


By the hypothesis of the theorem, we have $(m /(m+1))$ $I<Y_{1}, Y_{2}<I$, which implies that $m /(m+1)<$ $\lambda_{1 l}, \lambda_{2 j}<1 ; \quad l, j=1,2, \ldots, n$. Note that $\left(m t_{i} /\left(m t_{i}+\right.\right.$ $1))<(m /(m+1)) ; i=1,2, \ldots, m$. Then, $\left(m t_{i} /\left(m t_{i}+1\right)\right)$ $<\lambda_{1 l}, \lambda_{2 j}<1 ; l, j=1,2, \ldots, n ; i=1,2, \ldots, m$. Therefore, it follows (32) and (33) that

$$
\begin{aligned}
\left\|\left(C_{i}-D_{i}\right) B\right\| & =\max _{l, j}\left\{\frac{\sqrt{\left(1-\lambda_{1 l}\right)\left(1-\lambda_{2 j}\right)}\left(\lambda_{2 j}^{t_{i}}-\lambda_{1 l}^{t_{i}}\right)}{\left(\lambda_{2 j}-\lambda_{1 l}\right) \lambda_{2 j}^{\left(t_{i} / 2\right)} \lambda_{1 l}^{\left(t_{i} / 2\right)}}\right\} \\
& =\max _{l, j}\left\{f\left(\lambda_{1 l}, \lambda_{2 j}, t_{i}\right)\right\},
\end{aligned}
$$

where $f(x, y, t)$ is defined in Lemma 6 . It is easy to verify that

$$
\|E B\|=\max _{l, j}\left\{\frac{\sqrt{\left(1-\lambda_{1 l}\right)\left(1-\lambda_{2 j}\right)}}{\lambda_{2 j}^{(1 / 2)} \lambda_{1 l}^{(1 / 2)}}\right\}<\frac{1}{m} .
$$

A combination of Lemma 6, (36)-(38) gives that

$$
\left\|\sum_{i=(s+1)}^{m} J_{i}\left(C_{i}-D_{i}\right) B-\sum_{i=1}^{s} J_{i} E_{i} B\right\|<\frac{m-s}{m}+\frac{s}{m}=1,
$$

which implies $I+\sum_{i=(s+1)}^{m} J_{i}\left(C_{i}-D_{i}\right) B-\sum_{i=1}^{s} J_{i} E B$ is nonsingular. It follows (34) that vecW=0. Recall that $W=P_{2}\left(Y_{1}-Y_{2}\right) P_{1}^{*}$. Therefore, $Y_{1}=Y_{2}$, which means the HPD solution on $[(m /(m+1)) I, I]$ of $(1)$ is unique.

\section{The Homotopy Continuation Iterative Method}

In this section, by means of the homotopy continuation iterative method (see [34], for more details), we derive a numerical iterative process for solving the matrix equation (1).

Define the nonlinear map F: $\mathscr{P}^{n \times n} \subset \mathscr{C}^{n \times n} \longrightarrow \mathscr{C}^{n \times n}$ by

$$
F(X)=I-\sum_{i=1}^{s} B_{i}^{*} X^{-1} B_{i}-\sum_{i=s+1}^{m} B_{i}^{*} X^{t_{i}} B_{i} .
$$

Consider the homotopy $H:[0,1] \times \mathscr{P}^{n \times n} \longrightarrow \mathscr{P}^{n \times n}$ :

$$
H(t, X)=I-t \sum_{i=1}^{s} B_{i}^{*} X^{-1} B_{i}-t \sum_{i=s+1}^{m} B_{i}^{*} X^{t_{i}} B_{i}-X .
$$

Then, at $t=0$, the solution of $H(t, X)=0$ is a known matrix $I$, while at $t=1$, the solution $X$ of $H(t, X)=0$ also solves $F(X)=X$. To discuss the numerical method for solving the homotopy equation $H(t, X)=0$, we rewrite the homotopy equation $H(t, X)=0$ as the following fixed point form.

Assume that $G:[0,1] \times \mathscr{P}^{n \times n} \subset[0,1] \times \mathscr{C}^{n \times n} \longrightarrow \mathscr{P}^{n \times n}$ is a map such that

$$
X(t)=G(t, X(t)), \quad t \in[0,1],
$$

where $X:[0,1] \longrightarrow \mathscr{P}^{n \times n}$ denotes the solution of $H(t, X)=0$. Then, for each $t$, we can consider the iterative process:

$$
X_{n+1}=G\left(t, X_{n}\right) .
$$

Since for a fixed $t$, this process will converge to $X(t)$ only for starting values near that point; to overcome the local convergence of iterative process, we consider the following numerical continuation process.

A partition of $J=[0,1]$ :

$$
0=t_{0}<t_{1}<\cdots<t_{N}=1
$$

and a sequence of integers $\left\{j_{k}\right\}, k=1, \ldots, N-1$, is chosen with the property that the points

$$
\begin{cases}X_{k,(j+1)}=G\left(t_{k}, X_{k, j}\right), & j=0, \ldots, j_{k}-1, \quad k=1, \ldots, N-1, \\ X_{(k+1), 0}=X_{k, j_{k}}, & X_{1,0}=X_{0},\end{cases}
$$

are well-defined and such that

$$
X_{N,(j+1)}=G\left(1, X_{N, j}\right),
$$

converges to $X(1)$ as $j \longrightarrow \infty$.

The main idea is to choose partition (44) so that $X\left(t_{k}\right)$ lies in some domain of attraction $D_{t_{(k+1)}}$, for each $k, 1 \leq k \leq N$. Then, if $X_{k, 0} \in D_{t_{(k+1)}}$, the sequence generated by (43) for $t=$ $t_{k}$ must produce an iterate $X_{k, j_{k}} \in D_{t_{(k+1)}}$, which in turn can be taken as the starting point $X_{(k+1), 0}=X_{k, j_{k}}$ for the next iteration involving $t_{k+1}$. Thus, with $X_{1,0}=X_{0}$ as initial point, the entire process can be carried out until finally $t_{k}=t_{N}=1$ is reached. For $t=1, X_{N, 0}=X_{N, j_{(N-1)}}$ is then in $D_{1}$ which ensures that (45) converges to $X(1)$ as $j \longrightarrow \infty$.

To discuss the feasibility of the abovementioned numerical continuation process, we will use the following definition and lemmas which can be found in [34].

Definition 1 (see [34]). If a partition (44) exists so that with some sequence of integers $\left\{j_{k}\right\}$, the entire process (45)-(46) is well defined so that (46) converges to $X(1)$, and then the numerical continuation process $(45)-(46)$ is called feasible.

Definition 2 (see [34]). Let $G: D \subset R^{n} \longrightarrow R^{n}$ be a given mapping. Then, any nonempty set $D_{0} \subset D$ is a domain of attraction of the iterative process:

$$
x_{n+1}=G\left(x_{n}\right), \quad n=0,1, \ldots,
$$

with respect to the point $x_{*}$, if for any $x_{0} \in D_{0}$, we have $\left\{x_{n}\right\} \subset D$ and $\lim _{n \longrightarrow \infty} x_{n}=x_{*}$.

If $x_{*} \in \operatorname{int}\left(D_{0}\right)$ for some domain of attraction $D_{0}$, then $x_{*}$ is a point of attraction of (47).

Lemma 7 (see [34]). Let $G: D \subset R^{n} \longrightarrow R^{n}$ be Fréchet differentiable at the fixed point $x_{*} \in \operatorname{int}(D)$ of $G$. If $\rho\left(G \prime\left(x_{*}\right)\right)<1$, then $x_{*}$ is a point of attraction of (47) and, more precisely, there is an open ball $S\left(x_{*}, r\right)$ with center $x_{*}$ and radius $r>0$ which is a domain of attraction of (47) with respect to $x_{*}$. Here, $\rho(\cdot)$ denotes the spectral radius of $G^{\prime}\left(x_{*}\right)$. 
Lemma 8 (see [34]). Let $G:[0,1] \times D \subset[0,1] \times R^{n} \longrightarrow R^{n}$, where $D$ is open and assume that $x:[0,1] \longrightarrow D$ is continuous and satisfies $x(t)=G(t, x(t))$. Let $G$ have a Fréchet derivative with respect to $x$ at $(t, x(t))$ for every $t \in[0,1]$. If $G_{x}(t, x(t))$ is continuous on $[0,1] \times D$ and $\rho\left(G_{x}(t, x(t))\right)$ $<1$ for all $t \in[0,1]$, then the numerical continuation process (45)-(46) is feasible.

In what follows, we derive a sufficient condition for the existence of a unique HPD solution of the homotopy equation $H(t, X)=0$ for all $t \in[0,1]$.

Theorem 3. If $\lambda\left(\sum_{i=1}^{m} B_{i}^{*} B_{i}\right)<\left(m /(m+1)^{2}\right)$, then for arbitrary $t \in[0,1]$, the homotopy equation $H(t, X)=0$ has a unique HPD solution on $[(m /(m+1)) I, I]$.

Proof. Since $t>0$, then the homotopy equation $H(t, X)=0$ can be rewritten as

$$
X+\sum_{i=1}^{s}\left(\sqrt{t} B_{i}\right)^{*} X^{-1}\left(\sqrt{t} B_{i}\right)+\sum_{i=(s+1)}^{m}\left(\sqrt{t} B_{i}\right)^{*} X^{t_{i}}\left(\sqrt{t} B_{i}\right)=I .
$$

By the hypothesis of the theorem, we have

$$
\begin{aligned}
\lambda_{1}\left(\sum_{i=1}^{m}\left(\sqrt{t} B_{i}\right)^{*}\left(\sqrt{t} B_{i}\right)\right) & =\lambda_{1}\left(\sum_{i=1}^{m} t B_{i}^{*} B_{i}\right) \leq \lambda_{1}\left(\sum_{i=1}^{m} B_{i}^{*} B_{i}\right) \\
& <\frac{m}{(m+1)^{2}} .
\end{aligned}
$$

It follows from Theorem 2 that the homotopy equation $H(t, X)=0$ has a unique HPD solution on $[(m /(m+1)) I, I]$.

In the next theorem, the local convergence of the iterative process (43) is obtained.

Theorem 4. If $F(X)=X$ has a unique HPD solution $X_{*}$ on $((m /(m+1)) I, I)$, then there exists an open ball $N\left(X_{*}, \delta\right)$ with center $X_{*}$ and radius $\delta>0$ such that, for any starting value $X_{0} \in N\left(X_{*}, \delta\right), X_{n}=F\left(X_{n-1}\right)$ converges to $X_{*}$ as $n \longrightarrow \infty$.

Proof. Recall that $F(X)=I-\sum_{i=1}^{s} B_{i}^{*} X^{-1} B_{i}-\sum_{i=(s+1)}^{m} B_{i}^{*}$ $X^{t_{i}} B_{i}$. According to Lemma 2, for any $h \in \mathscr{P}^{n \times n}$, we have

$$
\begin{aligned}
F( & \left.X_{*}+h\right)-F\left(X_{*}\right) \\
= & \sum_{i=1}^{s} B_{i}^{*}\left(X_{*}^{-1}-\left(X_{*}+h\right)^{-1}\right) B_{i}+\sum_{i=s+1}^{m} B_{i}^{*}\left(X_{*}^{t_{i}}-\left(X_{*}+h\right)^{t_{i}}\right) B_{i} \\
= & \sum_{i=1}^{s} B_{i}^{*}\left(X_{*}+h\right)^{-1} h X_{*}^{-1} B_{i}-\sum_{i=s+1}^{m} \frac{B_{i}^{*} \sin t_{i} \pi}{\pi} \int_{0}^{\infty} h\left(\lambda I+X_{*}+h\right)^{-1} \lambda^{t_{i}-1} B_{i} \mathrm{~d} \lambda \\
& +\sum_{i=(s+1)}^{m} \frac{B_{i}^{*} \sin t_{i} \pi}{\pi} \int_{0}^{\infty} X_{*}\left(\lambda I+X_{*}\right)^{-1} h\left(\lambda I+X_{*}+h\right)^{-1} \lambda^{t_{i}-1} B_{i} \mathrm{~d} \lambda .
\end{aligned}
$$

By the definition of Fréchet derivative, we obtain

$$
\begin{aligned}
F^{\prime}\left(X_{*}\right) h= & \sum_{i=1}^{s} B_{i}^{*} X_{*}^{-1} h X_{*}^{-1} B_{i}-\sum_{i=(s+1)}^{m} \frac{B_{i}^{*} \sin t_{i} \pi}{\pi} \int_{0}^{\infty} h\left(\lambda I+X_{*}\right)^{-1} \lambda^{t_{i}-1} B_{i} \mathrm{~d} \lambda \\
& +\sum_{i=(s+1)}^{m} \frac{B_{i}^{*} \sin t_{i} \pi}{\pi} \int_{0}^{\infty} X_{*}\left(\lambda I+X_{*}\right)^{-1} h\left(\lambda I+X_{*}\right)^{-1} \lambda^{t_{i}-1} B_{i} \mathrm{~d} \lambda .
\end{aligned}
$$

Let $\lambda$ be any eigenvalue of $F^{\prime}\left(X_{*}\right)$. Then, there exists a nonzero matrix $h_{*}$ such that

$$
F^{\prime}\left(X_{*}\right) h_{*}=\lambda h_{*} \text {. }
$$

Since $X_{*}$ is the unique HPD solution of $F(X)=X$, then by Theorem 1 , there exists $P \in \mathcal{U}^{n \times n}, Q_{i} \in \mathscr{C}^{n \times n}$, $i=1,2, \ldots, m$, and diagonal matrices $\Gamma, \Lambda>0$ such that

$$
B_{i}= \begin{cases}P^{*} \Gamma^{(1 / 2)} Q_{i} \Lambda P, & i=1,2, \ldots, s, \\ P^{*} \Gamma^{-\left(t_{i} / 2\right)} Q_{i} \Lambda P, & i=s+1, s+2, \ldots, m,\end{cases}
$$

where

$$
\begin{aligned}
\sum_{i=1}^{m} Q_{i}^{*} Q_{i} & =I, \\
\Gamma+\Lambda^{2} & =I .
\end{aligned}
$$

In this case, $X_{*}=P^{*} \Gamma P$, where $\Gamma=\operatorname{diag}\left(\lambda_{1}, \lambda_{2}, \ldots, \lambda_{n}\right)$ with $\left\{\lambda_{j}\right\}$ the eigenvalues of $X_{*}$. Therefore, (52) can be rewritten as 


$$
\begin{aligned}
F_{\prime}\left(X_{*}\right) h_{*}= & \sum_{i=1}^{s} P^{*} \Lambda Q_{i}^{*} \Gamma^{-(1 / 2)} P h_{*} P^{*} \Gamma^{-(1 / 2)} Q_{i} \Lambda P \\
& -\sum_{i=(s+1)}^{m} \frac{\sin t_{i} \pi}{\pi} \int_{0}^{\infty} P^{*} \Lambda Q_{i}^{*} \Gamma^{\left(-t_{i} / 2\right)} P h_{*} P^{*}(\lambda I+\Gamma)^{-1} \lambda^{t_{i}-1} \Gamma^{\left(-t_{i} / 2\right)} Q_{i} \Lambda P \mathrm{~d} \lambda \\
& +\sum_{i=(s+1)}^{m} \frac{\sin t_{i} \pi}{\pi} \int_{0}^{\infty} P^{*} \Lambda Q_{i}^{*} \Gamma^{1-\left(t_{i} / 2\right)}(\lambda I+\Gamma)^{-1} P h_{*} P^{*}(\lambda I+\Gamma)^{-1} \lambda^{t_{i}-1} \Gamma^{\left(-t_{i} / 2\right)} Q_{i} \Lambda P \mathrm{~d} \lambda \\
= & \lambda h_{*} .
\end{aligned}
$$

Let $z=P h_{*} P^{*}$. It follows that

$$
\begin{aligned}
& \sum_{i=1}^{s} \Lambda Q_{i}^{*} \Gamma^{-(1 / 2)} z \Gamma^{-(1 / 2)} Q_{i} \Lambda-\sum_{i=(s+1)}^{m} \frac{\sin t_{i} \pi}{\pi} \int_{0}^{\infty} \Lambda Q_{i}^{*} \Gamma^{\left(-t_{i} / 2\right)} z(\lambda I+\Gamma)^{-1} \lambda^{t_{i}-1} \Gamma^{\left(-t_{i} / 2\right)} Q_{i} \Lambda \mathrm{d} \lambda \\
& \quad+\sum_{i=(s+1)}^{m} \frac{\sin t_{i} \pi}{\pi} \int_{0}^{\infty} \Lambda Q_{i}^{*} \Gamma^{1-\left(t_{i} / 2\right)}(\lambda I+\Gamma)^{-1} z(\lambda I+\Gamma)^{-1} \lambda^{t_{i}-1} \Gamma^{\left(-t_{i} / 2\right)} Q_{i} \Lambda \mathrm{d} \lambda=\lambda z .
\end{aligned}
$$

Define the operator $\mathscr{L}: \mathscr{C}^{n \times n} \longrightarrow \mathscr{C}^{n \times n}$ by

$$
\begin{aligned}
\mathscr{L} z= & \sum_{i=1}^{s} \Lambda Q_{i}^{*} \Gamma^{-(1 / 2)} z \Gamma^{-(1 / 2)} Q_{i} \Lambda-\sum_{i=(s+1)}^{m} \frac{\sin t_{i} \pi}{\pi} \int_{0}^{\infty} \Lambda Q_{i}^{*} \Gamma^{\left(-t_{i} / 2\right)} z(\lambda I+\Gamma)^{-1} \Gamma^{\left(-t_{i} / 2\right)} Q_{i} \Lambda \lambda^{t_{i}-1} \mathrm{~d} \lambda \\
& +\sum_{i=(s+1)}^{m} \frac{\sin t_{i} \pi}{\pi} \int_{0}^{\infty} \Lambda Q_{i}^{*} \Gamma^{1-\left(t_{i} / 2\right)}(\lambda I+\Gamma)^{-1} z(\lambda I+\Gamma)^{-1} \Gamma^{\left(-t_{i} / 2\right)} Q_{i} \Lambda \lambda^{t_{i}-1} \mathrm{~d} \lambda .
\end{aligned}
$$

Then,

$$
\mathscr{L} z=\lambda z .
$$

$$
\begin{aligned}
\operatorname{vec}(\mathscr{L} z)= & \sum_{i=1}^{s}\left[\Gamma^{-(1 / 2)} Q_{i} \Lambda\right]^{T} \otimes\left[\Lambda U_{i}^{*} \Gamma^{-(1 / 2)}\right] \cdot \operatorname{vec} z \\
& -\sum_{i=(s+1)}^{m} \frac{\sin t_{i} \pi}{\pi} \int_{0}^{\infty}\left[(\lambda I+\Gamma)^{-1} \Gamma^{-\left(t_{i} / 2\right)} Q_{i} \Lambda\right]^{T} \otimes\left(\Lambda Q_{i}^{*} \Gamma^{-\left(t_{i} / 2\right)}\right) \lambda^{t_{i}-1} \mathrm{~d} \lambda \cdot \operatorname{vec} z \\
& +\sum_{i=(s+1)}^{m} \frac{\sin t_{i} \pi}{\pi} \int_{0}^{\infty}\left[(\lambda I+\Gamma)^{-1} \Gamma^{-\left(t_{i} / 2\right)} Q_{i} \Lambda\right]^{T} \otimes\left[\Lambda Q_{i}^{*} \Gamma^{1-\left(t_{i} / 2\right)}(\lambda I+\Gamma)^{-1}\right] \lambda^{t_{i}-1} \mathrm{~d} \lambda \cdot \operatorname{vec} z \\
= & \sum_{i=1}^{s}(\Lambda \otimes \Lambda)\left(Q_{i}^{T} \otimes U_{i}^{*}\right)\left(\Gamma^{(1 / 2)} \otimes \Gamma^{(1 / 2)}\right) \cdot \operatorname{vec} z \\
& -\sum_{i=(s+1)}^{m} \frac{\sin t_{i} \pi}{\pi}(\Lambda \otimes \Lambda)\left(Q_{i}^{T} \otimes Q_{i}^{*}\right)\left(\Gamma^{\left(-t_{i} / 2\right)} \otimes \Gamma^{-\left(t_{i} / 2\right)}\right) \int_{0}^{\infty}\left[(\lambda I+\Gamma)^{-1} \otimes I\right] \lambda^{t_{i}-1} \mathrm{~d} \lambda \cdot \operatorname{vec} z \\
& +\sum_{i=(s+1)}^{m} \frac{\sin t_{i} \pi}{\pi}(\Lambda \otimes \Lambda)\left(Q_{i}^{T} \otimes Q_{i}^{*}\right)\left(\Gamma^{-\left(t_{i} / 2\right)} \otimes \Gamma^{1-\left(t_{i} / 2\right)}\right) \int_{0}^{\infty}(\lambda I+\Gamma)^{-1} \otimes(\lambda I+\Gamma)^{-1} \lambda^{t_{i}-1} \mathrm{~d} \lambda \operatorname{vec} z \\
= & \lambda \operatorname{vec} z .
\end{aligned}
$$


Let

$$
\begin{aligned}
B & =\Lambda \otimes \Lambda, J_{i}=Q_{i}^{T} \otimes Q_{i}^{*}, \\
C_{i} & =\left(\Gamma^{\left(-t_{i} / 2\right)} \otimes \Gamma^{\left(-t_{i} / 2\right)}\right) \cdot \frac{\sin t_{i} \pi}{\pi} \int_{0}^{\infty}\left[(\lambda I+\Gamma)^{-1} \otimes I\right] \lambda^{t_{i}-1} \mathrm{~d} \lambda, \quad i=s+1, s+2, \ldots, m, \\
D_{i} & =\left(\Gamma^{\left(-t_{i} / 2\right)} \otimes \Gamma^{\left(1-\left(t_{i} / 2\right)\right)}\right) \cdot \frac{\sin t_{i} \pi}{\pi} \int_{0}^{\infty}(\lambda I+\Gamma)^{-1} \otimes(\lambda I+\Gamma)^{-1} \lambda^{t_{i}-1} \mathrm{~d} \lambda, \quad i=s+1, s+2, \ldots, m, \\
E & =\Gamma^{-(1 / 2)} \otimes \Gamma^{-(1 / 2)}=\operatorname{diag}\left(\lambda_{l}^{-(1 / 2)} \cdot \lambda_{j}^{-(1 / 2)}\right)_{n^{2} \times n^{2}} ; \quad l, j=1,2, \ldots, n .
\end{aligned}
$$

Then, (59) can be rewritten as According to (36), (55), (57), (59), and (61), we have that $\operatorname{vec}(\mathscr{L} z)=\left[\sum_{i=s+1}^{m} B J_{i}\left(D_{i}-C_{i}\right)+\sum_{i=1}^{s} B J_{i} E\right] \cdot \operatorname{vec} z=\lambda \cdot \operatorname{vec} z$.

$$
\begin{aligned}
\rho\left(F^{\prime}\left(X_{*}\right)\right) & =\max \{|\lambda|\}=\rho\left(\sum_{i=s+1}^{m} B J_{i}\left(D_{i}-C_{i}\right)+\sum_{i=1}^{s} B J_{i} E\right) \\
& \leq\left\|\sum_{i=(s+1)}^{m} B J_{i}\left(D_{i}-C_{i}\right)+\sum_{i=1}^{s} B J_{i} E\right\| \leq \sum_{i=(s+1)}^{m}\left\|B\left(C_{i}-D_{i}\right)\right\|+\sum_{i=1}^{s}\|B E\| .
\end{aligned}
$$

Assume that

$$
0<\sigma_{j}=\sqrt{1-\lambda_{j}}, \quad j=1,2, \ldots, n .
$$

$$
\Lambda=\operatorname{diag}\left(\sigma_{1}, \sigma_{2}, \ldots, \sigma_{n}\right) .
$$

According to Lemma 5 and (64), we have

By (54), we have

$$
\begin{aligned}
B & =\operatorname{diag}\left(\sigma_{l} \cdot \sigma_{j}\right)_{n^{2} \times n^{2}}=\operatorname{diag}\left(\sqrt{1-\lambda_{l}} \cdot \sqrt{1-\lambda_{j}}\right)_{n^{2} \times n^{2}} \\
C_{i} & =\left(\Gamma^{-\left(t_{i} / 2\right)} \otimes \Gamma^{-\left(t_{i} / 2\right)}\right) \cdot \frac{\sin t_{i} \pi}{\pi} \int_{0}^{\infty}\left[(\lambda I+\Gamma)^{-1} \otimes I\right] \lambda^{t_{i}-1} \mathrm{~d} \lambda \\
& =\operatorname{diag}\left(\lambda_{l}^{-\left(t_{i} / 2\right)} \cdot \lambda_{j}^{-\left(t_{i} / 2\right)} \cdot \frac{\sin t_{i} \pi}{\pi} \int_{0}^{\infty}\left(\lambda+\lambda_{l}\right)^{-1} \lambda^{t_{i}-1} \mathrm{~d} \lambda\right)_{n^{2} \times n^{2}} \\
& =\operatorname{diag}\left(\lambda_{l}^{-\left(t_{i} / 2\right)} \cdot \lambda_{j}^{-\left(t_{i} / 2\right)} \cdot \lambda_{l}^{t_{i}-1}\right)_{n^{2} \times n^{2}} \\
& =\operatorname{diag}\left(\lambda_{l}^{\left(t_{i} / 2\right)-1} \cdot \lambda_{j}^{-\left(t_{i} / 2\right)}\right)_{n^{2} \times n^{2}}, i=(s+1),(s+2), \ldots, m, l, j=1,2, \ldots, n, \\
D_{i} & =\left(\Gamma^{-\left(t_{i} / 2\right)} \otimes \Gamma^{1-\left(t_{i} / 2\right)}\right) \cdot \frac{\sin t_{i} \pi}{\pi} \int_{0}^{\infty}(\lambda I+\Gamma)^{-1} \otimes(\lambda I+\Gamma)^{-1} \lambda^{t_{i}-1} \mathrm{~d} \lambda \\
& =\operatorname{diag}\left(\lambda_{l}^{-\left(t_{i} / 2\right)} \cdot \lambda_{j}^{1-\left(t_{i} / 2\right)} \cdot \frac{\sin t_{i} \pi}{\pi} \int_{0}^{\infty}\left(\lambda+\lambda_{l}\right)^{-1}\left(\lambda+\lambda_{j}\right)^{-1} \lambda^{t_{i}-1} \mathrm{~d} \lambda\right)_{n^{2} \times n^{2}} \\
& =\operatorname{diag}\left(\frac{\lambda_{l}^{-\left(t_{i} / 2\right)} \cdot \lambda_{j}^{1-\left(t_{i} / 2\right)}\left(\lambda_{l}^{t_{i}-1}-\lambda_{j}^{t_{i}-1}\right)}{\lambda_{j}-\lambda_{l}}\right)_{n^{2} \times n^{2}}, i=(s+1),(s+2), \ldots, m, l, j=1,2, \ldots, n .
\end{aligned}
$$


Since $(m /(m+1)) I<X_{*}<I$, then $\left(m t_{i} /\left(m t_{i}+1\right)\right)<(m /$ $(m+1))<\lambda_{l}, \lambda_{j}<1, \quad i=1,2, \ldots, m, l, j=1,2, \ldots, n$. Ву (65)-(67), we have that

$$
\begin{aligned}
\left\|\left(C_{i}-D_{i}\right) B\right\| & =\max _{l, j}\left\{\frac{\sqrt{\left(1-\lambda_{l}\right)\left(1-\lambda_{j}\right)}\left(\lambda_{j}^{t_{i}}-\lambda_{l}^{t_{i}}\right)}{\left(\lambda_{j}-\lambda_{l}\right) \lambda_{j}^{\left(t_{i} / 2\right)} \lambda_{l}^{\left(t_{i} / 2\right)}}\right\}=\max _{l, j}\left\{f\left(\lambda_{l}, \lambda_{j}, t_{i}\right)\right\} \\
\left\|E_{i} B\right\| & =\max _{l, j}\left\{\frac{\sqrt{\left(1-\lambda_{l}\right)\left(1-\lambda_{j}\right)}}{\lambda_{j}^{\left(t_{i} / 2\right)} \lambda_{l}^{\left(t_{i} / 2\right)}}\right\},
\end{aligned}
$$

where $f(x, y, t)$ is defined in Lemma 6. Combining Lemma 6 (62) with (68) gives that

$$
\rho\left(F^{\prime}\left(X_{*}\right)\right) \leq \sum_{i=(s+1)}^{m}\left\|B\left(C_{i}-D_{i}\right)\right\|+\sum_{i=1}^{s}\|B E\|<1 .
$$

According to Lemma 7, there exists an open ball $N\left(X_{*}, \delta\right)$ with center $X_{*}$ and radius $\delta>0$ such that, for any starting value $X_{0} \in N\left(X_{*}, \delta\right), X_{n}=F\left(X_{n-1}\right)$ converges to $X_{*}$ as $n \longrightarrow \infty$.

In the next theorem, we will prove the numerical continuation process $(45)-(46)$ is feasible.
Theorem 5. If $\lambda_{1}\left(\sum_{i=1}^{m} B_{i}^{*} B_{i}\right)<m /(m+1)^{2}$, then the numerical continuation process (45)-(46) is feasible.

Proof. Define the map G: $[0,1] \times \mathscr{P}^{n \times n} \longrightarrow \mathscr{P}^{n \times n}$ by

$$
G(t, X(t))=I-t \sum_{i=1}^{s} B_{i}^{*} X^{-1} B_{i}-t \sum_{i=(s+1)}^{m} B_{i}^{*} X^{t_{i}} B_{i}
$$

In the following, we will prove the numerical continuation processes (45)-(46) are feasible.

By Lemma 2, for any $h \in \mathscr{P}^{n \times n}$, we have

$$
\begin{gathered}
G(t, X(t)+h)-G(t, X(t)), \\
G(t, X(t)+h)-G(t, X(t)) \\
=t \sum_{i=1}^{s} B_{i}^{*}\left(X^{-1}-(X+h)^{-1}\right) B_{i}+t \sum_{i=(s+1)}^{m} B_{i}^{*}\left(X^{t_{i}}-(X+h)^{t_{i}}\right) B_{i} \\
=t \sum_{i=1}^{s} B_{i}^{*} X^{-1} h(X+h)^{-1} B_{i}-t \sum_{i=(s+1)}^{m} \frac{B_{i}^{*} \sin t_{i} \pi}{\pi} \int_{0}^{\infty} h(\lambda I+X+h)^{-1} \lambda^{t_{i}-1} B_{i} \mathrm{~d} \lambda \\
+t \sum_{i=(s+1)}^{m} \frac{B_{i}^{*} \sin t_{i} \pi}{\pi} \int_{0}^{\infty} X(\lambda I+X)^{-1} h(\lambda I+X+h)^{-1} \lambda^{t_{i}-1} B_{i} \mathrm{~d} \lambda .
\end{gathered}
$$

By the definition of Fréchet derivative, we obtain

$$
\begin{aligned}
G^{\prime}(t, X(t)) h= & t \sum_{i=1}^{s} B_{i}^{*} X^{-1} h X^{-1} B_{i}-t \sum_{i=(s+1)}^{m} \frac{B_{i}^{*} \sin t_{i} \pi}{\pi} \int_{0}^{\infty} h(\lambda I+X)^{-1} \lambda^{t_{i}-1} B_{i} \mathrm{~d} \lambda \\
& +t \sum_{i=(s+1)}^{m} \frac{B_{i}^{*} \sin t_{i} \pi}{\pi} \int_{0}^{\infty} X(\lambda I+X)^{-1} h(\lambda I+X)^{-1} \lambda^{t_{i}-1} B_{i} \mathrm{~d} \lambda .
\end{aligned}
$$

Using the same technique described in Theorem 4, we have that

$$
\rho\left(G^{\prime}(t, X(t))\right)<t\left(\sum_{i=1}^{s} \max _{l, j}\left\{\frac{\sqrt{\left(1-\lambda_{l}\right)\left(1-\lambda_{j}\right)}}{\lambda_{j}^{(1 / 2)} \lambda_{l}^{(1 / 2)}}\right\}+\sum_{i=(s+1)}^{m} \max _{l, j}\left\{\frac{\sqrt{1-\lambda_{l}} \sqrt{1-\lambda_{j}}\left(\lambda_{j}^{t_{i}}-\lambda_{l}^{t_{i}}\right)}{\left(\lambda_{j}-\lambda_{l}\right) \lambda_{l}^{\left(t_{i} / 2\right)} \lambda_{j}^{\left(t_{i} / 2\right)}}\right\}\right)<1,
$$


where $\quad\left(m t_{i} /\left(m t_{i}+1\right)\right)<(m /(m+1))<\lambda_{l}, \lambda_{j}<1, \quad l, j=$ $1,2, \ldots, n$.

According to Lemma 2, the numerical continuation processes (45)-(46) are feasible.

\section{Conclusions}

We have introduced a class of nonlinear matrix equations that is wider than those studied earlier in the literature. We have derived some sufficient and necessary conditions for the existence and uniqueness of HPD solution of (1). In order to apply the homotopy continuation iterative method proposed by Avila [34] to the nonlinear matrix equation (1), we have constructed a related homotopy matrix equation $H(t, X)=0$ and have derived a sufficient condition for the existence of a unique HPD solution of this equation. We have cited the definition and the judgment theorem of feasible for the numerical continuation process (45)-(46), which was proposed in [34]. And then we have derived the condition for feasible of the numerical continuation processes (45)-(46). Furthermore, we obtained the feasible conclusion of the numerical method by verifying the conditions in Theorem 2.5 in [34].

\section{Data Availability}

No data were used to support our study.

\section{Conflicts of Interest}

The authors declare that there are no conflicts of interest regarding the publication of this article.

\section{Authors' Contributions}

All authors read and approved the final manuscript.

\section{Acknowledgments}

This work was supported in part by the National Nature Science Foundation of China (11601277).

\section{References}

[1] W. N. Anderson, G. B. Kleindorfer, P. R. Kleindorfer, and M. B. Woodroofe, "Consistent estimates of the parameters of a linear system," The Annals of Mathematical Statistics, vol. 40, no. 6, pp. 2064-2075, 1969.

[2] B. L. Buzbee, G. H. Golub, and C. W. Nielson, "On direct methods for solving Poisson's equations," SIAM Journal on Numerical Analysis, vol. 7, no. 4, pp. 627-656, 1970.

[3] R. S. Bucy, "A priori bounds for the Riccati equation," in Proceedings of the Berkley Symposium on Mathematical Statistics and Probability, vol. III, pp. 645-656, Probability Theory, Univ. of California Press, Berkeley, CA, USA, 1972.

[4] J. Zabezyk, "Remarks on the control of discrete time distributed parameter systems," SIAM Journal on Control and Optimization, vol. 12, pp. 721-735, 1974.

[5] W. Pusz and S. L. Woronowicz, "Functional calculus for sesquilinear forms and the purification map," Reports on Mathematical Physics, vol. 8, no. 2, pp. 159-170, 1975.
[6] D. V. Ouellette, "Schur complements and statistics," Linear Algebra and Its Applications, vol. 36, pp. 187-295, 1981.

[7] W. N. Anderson, T. D. Morley, and G. E. Trapp, "The cascade limit, the shorted operator and quadratic optimal control," in Linear Circuits, Systems and Signal Processsing: Theory and Application, C. I. Byrnes, C. F. Martin, and R. E. Saeks, Eds., pp. 3-7, North-Holland, New York, NY, USA, 1988.

[8] C.-H. Guo and W.-W. Lin, "The matrix equation $X+A T X^{-1} A=Q$ and its application in nano research," SIAM Journal on Scientific Computing, vol. 32, no. 5, pp. 3020-3038, 2010.

[9] J. C. Engwerda, "On the existence of a positive definite solution of the matrix equation $X+A^{\mathrm{T}} X^{-1} A=I$," Linear Algebra and Its Applications, vol. 194, pp. 91-108, 1993.

[10] C.-H. Guo and P. Lancaster, "Iterative solution of two matrix equations," Mathematics of Computation, vol. 68, no. 228, pp. 1589-1604, 1999.

[11] J.-g. Sun and S.-F. Xu, "Perturbation analysis of the maximal solution of the matrix equation $X+A^{*} X^{-1} A=P$. II," Linear Algebra and Its Applications, vol. 362, pp. 211-228, 2003.

[12] V. I. Hasanov and I. G. Ivanov, "On two perturbation estimates of the extreme solutions to the equations $X \pm A^{*} X^{-1} A=Q$," Linear Algebra and Its Applications, vol. 413, no. 1, pp. 81-92, 2006.

[13] X.-G. Liu and H. Gao, "On the positive definite solutions of the matrix equations $X^{s} \pm A^{\mathrm{T}} X^{-\mathrm{t}} A=I_{\mathrm{n}}$," Linear Algebra and Its Applications, vol. 368, pp. 83-97, 2003.

[14] V. I. Hasanov and S. M. El-Sayed, "On the positive definite solutions of nonlinear matrix equation $X+A^{*} X^{-\delta} A=Q$," Linear Algebra and Its Applications, vol. 412, no. 2-3, pp. 154-160, 2006.

[15] X. Yin, S. Liu, and T. Li, "On positive definite solutions of the matrix equation $X+A^{*} X^{-\mathrm{q}} A=Q(0<\mathrm{q} \leq 1)$," Taiwanese Journal of Mathematics, vol. 16, no. 4, pp. 1391-1407, 2012.

[16] M. H. Wang, M. S. Wei, and S. R. Hu, "The extremal solution of the matrix equation $X^{s}+A^{*} X^{-q} A=I$." Applied Mathematics and Computation, vol. 220, pp. 193-199, 2013.

[17] D. M. Zhou, G. L. Chen, and X. Y. Zhang, "Some inequalities for the nonlinear matrix equation $X^{s}+A^{*} X^{-t} A=Q$ : trace, determinant and eigenvalue," Applied Mathematics and Computation, vol. 224, pp. 21-28, 2013.

[18] G.-F. Zhang, W.-W. Xie, and J.-Y. Zhao, "Positive definite solutions of the nonlinear matrix equation $X+A^{*} X^{q} A=Q(q>0)$," Applied Mathematics and Computation, vol. 217, no. 22, pp. 9182-9188, 2011.

[19] Y.-M. He and J.-H. Long, "On the Hermitian positive definite solution of the nonlinear matrix equation," Applied Mathematics and Computation, vol. 216, no. 12, pp. 3480-3485, 2010.

[20] X. Duan, C. Li, and A. Liao, "Solutions and perturbation analysis for the nonlinear matrix equation," Applied Mathematics and Computation, vol. 218, no. 8, pp. 4458-4466, 2011.

[21] A. M. Sarhan, N. M. El-Shazly, and E. M. Shehata, "On the existence of extremal positive definite solutions of the nonlinear matrix equation $X^{r}+\sum_{i=1}^{m} A_{i}^{*} X^{\delta_{i}} A_{i}=I$." Mathematical and Computer Modelling, vol. 51, pp. 1107-1117, 2010.

[22] A. J. Liu and G. L. Chen, "On the Hermitian positive definite solutions of nonlinear matrix equation $X^{s}+\sum_{i=1}^{m} A_{i}^{*} X^{-t_{i}} A_{i}=$ Q." Applied Mathematics and Computation, vol. 243, pp. 950-959, 2014.

[23] X. Yin, R. Wen, and L. Fang, "On the nonlinear matrix equation $X+\sum_{i=1}^{m} A_{i}^{*} X^{-q} A_{i}=Q(0<q \leq 1)$." Bulletin of the Korean Mathematical Society, vol. 51, no. 3, pp. 739-763, 2014. 
[24] J. Li and Y. H. Zhang, "The investigation on two kinds of nonlinear matrix equations," Bulletin of the Malaysian Mathematical Sciences Society, vol. 42, pp. 3323-3341, 2019.

[25] S. M. El-Sayed and A. C. M. Ran, "On an iteration method for solving a class of nonlinear matrix equaitons," SIAM Journal on Matrix Analysis and Applications, vol. 23, no. 3, pp. 632-645, 2002.

[26] A. C. M. Ran and M. C. B. Reurings, "On the nonlinear matrix equation $X+A^{*} \mathscr{F}(X) A=Q$ : solutions and perturvation theory," Linear Algebra and Its Applications, vol. 346, pp. 15-26, 2002.

[27] D. M. Zhou, G. L. Chen, G. X. Wu, and X. Y. Zhang, "On the nonlinear matrix equation $X^{s}+A^{*} F(X) A=Q$ with, $s \geq 1$." Journal of Computational Mathematics, vol. 31, no. 2, pp. 209-220, 2013.

[28] A. C. M. Ran and M. C. B. Reurings, "A fixed point theorem in partially ordered sets and some applications to matrix equations," Proceedings of the American Mathematical Society, vol. 132, no. 5, pp. 1435-1443, 2004.

[29] A. C. M. Ran, M. C. B. Reurings, and L. Rodman, "A perturbation analysis for nonlinear selfadjoint operator equations," SIAM Journal on Matrix Analysis and Applications, vol. 28, no. 1, pp. 89-104, 2006.

[30] M. M. Rashidi and A. M. Siddiqui, "Mostafa Asadi Application of homotopy analysis method to the unsteady squeezing flow of a second-grade fluid between circular plates," Mathematical Problems in Engineering, vol. 2010, Article ID 706840, 18 pages, 2010.

[31] V. Marinca and N. Herisanu, "On the flow of a Walters-type B viscoelastic fluid in a vertical channel with porous wall," International Journal of Heat and Mass Transfer, vol. 79, pp. 146-165, 2014.

[32] V. Marinca and N. Herisanua, "An optimal homotopy perturbation approach to thin film flow of a fourth grade fluid," AIP Conference Proceedings, vol. 1479, pp. 2383-2386, 2012.

[33] Z. Odibat and S. Kumar, "A robust computational algorithm of homotopy asymptotic method for solving systems of fractional differential equation," Journal of Computational and Nonlinear Dynamics, vol. 14, no. 8, Article ID 081004, 2019.

[34] J. H. Avila, "The feasibility of continuation methods for nonlinear equations," SIAM Journal on Numerical Analysis, vol. 11, no. 1, pp. 102-122, 1974.

[35] R. Bhatia, Matrix Analysis, Springer-Verlag, New York, NY, USA, 1997.

[36] S. F. Xu, Matrix Computation from Control System, Higher Education Press, Beijing, China, 2011.

[37] F. Z. Zhang, Matrix Theory: Basic Results and Techniques, Springer, Berlin, Germany, 1999. 